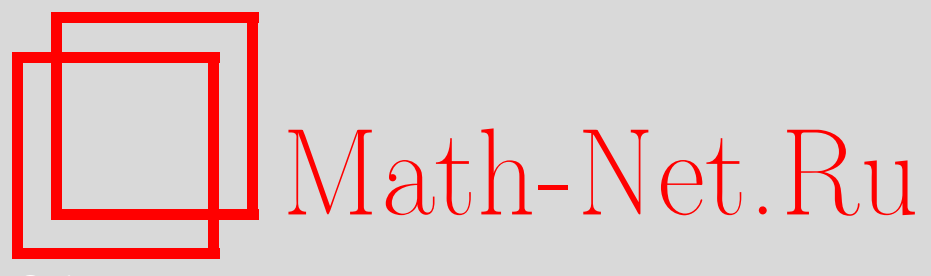

Д. В. Прохоренко, $A B C$-формула и $R$-операция для квантовых процессов с нестабильными состояниями, TMФ, 2006, том 149, номер 2, 183-201

DOI: https://doi.org/10.4213/tmf4226

Использование Общероссийского математического портала Math-Net.Ru подразумевает, что вы прочитали и согласны с пользовательским соглашением http://www.mathnet.ru/rus/agreement

Параметры загрузки:

IP : 54.197 .217 .227

26 апреля 2023 г., 16:42:25

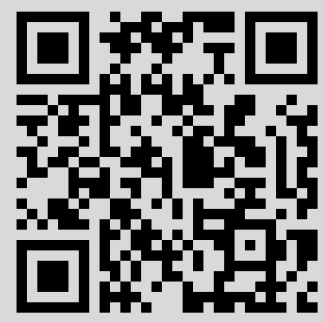




\title{
ABC-ФОРМУЛА И $R$-ОПЕРАЦИЯ ДЛЯ КВАНТОВЫХ ПРОЦЕССОВ С НЕСТАБИЛЬНЫМИ СОСТОЯНИЯМИ
}

\begin{abstract}
Для широкого класса гамильтонианов, используемых в квантовой теории поля и статистической физике, получена явная формула, описывающая поведение вакуумного среднего оператора эволюции на больших, но конечных интервалах времени. Эта формула справедлива также для процессов с нестабильными состояниями.
\end{abstract}

Ключевые слова: $R$-операция, процессы с нестабильными состояниями, квантовые динамические системы.

\section{1. ВВЕДЕНИЕ}

$S$-матрица, которая описывает поведение системы на бесконечных интервалах времени [1], [2], является важным объектом в квантовой теории поля. Однако существует большое количество важных задач (в частности, в квантовой оптике [3], в статистической физике [4], при изучении распадов в квантовой механике [5] и теории элементарных частиц [6]), в которых исследуется поведение системы на больших, но конечных интервалах времени. Общим методом для изучения подобных проблем является метод стохастического предела, развитый в работах Аккарди, Лу, Воловича и других (см., например, [7]). Основная идея этого метода состоит в получении и исследовании квантовых стохастических уравнений, описывающих поведение оператора эволюции при малых константах связи $\lambda$ и больших временах $t$. Поправки высших порядков к результатам, полученным методом стохастического предела, найдены в работе [8].

Мы будем рассматривать гамильтонианы в фоковском пространстве вида

$$
H=H_{0}+\lambda V,
$$

* Математический институт им. В. А. Стеклова РАН, Москва, Россия. E-mail: prokhor@mi.ras.ru 
где $H_{0}$ - свободный гамильтониан, а $V$ - взаимодействие, которое есть сумма мономов Вика с ядрами, лежащими в пространстве Шварца. Отметим, что мы рассматриваем трансляционно-неинвариантные гамильтонианы. Используя теорию возмущений, мы получим следующую явную формулу для вакуумного среднего оператора эволюции:

$$
\langle 0|U(t)| 0\rangle=e^{A t+B+C(t)},
$$

где $A$ и $B$ - константы, а $C(t) \rightarrow 0$ при $t \rightarrow \infty$. Эта формула (так называемая $A B C$-формула) получена в работе [9] двумя способами: с использованием волновых операторов и теории возмущений. Метод, основанный на использовании волновых операторов, позволяет установить эту формулу только в случае отсутствия распада. Метод, основанный на использовании теории возмущений, позволяет рассматривать также случай распада. Однако выражения для $A, B$ и $C(t)$, полученные в [9] методом теории возмущений, в случае распада требуют модификации, что и сделано в данной работе. Заметим, что формула получена для вакуумного среднего оператора эволюции, однако ее можно обобщить на случай матричных элементов между экспоненциальными векторами.

Интересно обобщение этого результата на случай трансляционно-инвариантных гамильтонианов, однако такая важная в квантовой оптике система, как атом, взаимодействующий с полем излучения, описывается трансляционно-неинвариантным гамильтонианом, к тому же в этой системе возбужденное состояние атома нестабильно (см. [3]).

Заметим, что для доказательства того, что интегралы, представляющие коэффициенты $A$ и $B$, существуют, мы будем использовать технику, подобную той, которая используется в теории перенормировок [1], [10], [11].

Асимптотическое поведение оператора эволюции при больших временах для системы заряженных частиц было исследовано в работе [12]. Отделение эффектов самодействия от эффектов рассеяния для трансляционно-инвариантных гамильтонианов, что необходимо для построения волновых операторов, было выполнено в [13] (о теории рассеяния для моделей Ли см. [14]).

В кинетической теории газов для определения коэффициентов переноса используется вириальное разложение. Было обнаружено, что это разложение содержит расходимости [15]. Эти расходимости возникают при изучении динамики конечного множества молекул при больших временах. Возможно, и здесь использование техники теории перенормировок окажется полезным.

Проблема рассеяния элементарных частиц является сложной, поскольку большинство элементарных частиц нестабильны [6]. Стабильная частица определяет неприводимое унитарное представление группы Пуанкаре. Представления группы Пуанкаре, соответствующие нестабильным частицам, рассмотрены в [16]. Новые перенормировочные предписания для волновой функции для нестабильных частиц 
предложены в работе [17], где также было показано, что обычные перенормировочные предписания для волновой функции в случае нестабильных частиц ведут к калибровочно-неинвариантному результату.

Золотое правило Ферми и экспоненциальное поведение при больших временах для одной модели, описывающей нестабильные частицы, исследовались в [18]. В работе [19] было показано, что нестабильную частицу можно рассматривать как открытую квантовую систему.

Возможно $A B C$-формулу можно использовать при построении волновых операторов в случае распада.

Работа построена следующим образом. В разделе 2 определен класс рассматриваемых гамильтонианов, в разделе 3 найдены достаточные условия существования коэффициента $A$, а в разделе 4 - достаточные условия существования коэффициента $B$.

\section{2. ОСНОВНАЯ ТЕОРЕМА}

Пусть $\mathcal{F}=L^{2}\left(\mathbb{R}^{d}\right)$ обозначает бозонное пространство Фока над одночастичным гильбертовым пространством $L^{2}\left(\mathbb{R}^{d}\right)$. Пусть $a^{+}(k)$ и $a(k)$ - операторы рождения и уничтожения в $\mathcal{F}$ :

$$
\left[a(k), a\left(k^{\prime}\right)\right]=\left[a^{+}(k), a^{+}\left(k^{\prime}\right)\right]=0, \quad\left[a(k), a^{+}\left(k^{\prime}\right)\right]=\delta\left(k-k^{\prime}\right) .
$$

Вакуумное состояние определяется соотношением $a(k)|0\rangle=0$. Рассмотрим в пространстве Фока $\mathcal{F}$ гамильтониан

$$
\begin{aligned}
H=\int & \omega(k) a^{+}(k) a(k) d k+\lambda \sum_{m, n=0}^{N} \int v_{m, n}\left(p_{1}, \ldots, p_{m} \mid p_{1}, \ldots, p_{n}\right) \times \\
& \times \prod_{i=1}^{m} a^{+}\left(p_{i}\right) d p_{i} \prod_{j=1}^{n} a\left(q_{j}\right) d q_{j}, \quad k, p_{i}, q_{j} \in \mathbb{R}^{d}, \quad N=1,2, \ldots,
\end{aligned}
$$

где $v_{m, n}-$ функции из пространства Шварца, $v_{0,0}=0$.

Мы рассматриваем следующий закон дисперсии:

$$
\omega(k)=\frac{k^{2}}{2}-\omega_{0}, \quad \omega_{0} \in \mathbb{R}, \quad \omega_{0} \neq 0,
$$

но основные результаты могут быть обобщены на релятивистский случай.

Определим оператор эволюции следующим образом:

$$
U(t):=e^{i t H_{0}} e^{-i t H}, \quad t \in \mathbb{R} .
$$

По определению [10], [20] граф Фридрихса - это четверка $\left(V^{\prime}, R, l^{+}, l^{-}\right)$, где $V^{\prime}-$ конечное упорядоченное множество, называемое множеством вершин, $R$ - конечное множество, называемое множеством ребер, $l^{+}$и $l^{-}$суть отображения $l^{ \pm}: R \rightarrow V^{\prime}$ такие, что $l^{+}(r)>l^{-}(r) \forall r \in R$. 
Обозначим через $V$ множество всех вершин, за исключением минимальной.

Граф Фридрихса называется связным, если для любых двух вершин $v \neq v^{\prime}$ существует чередующаяся последовательность вершин и ребер $v=v_{0}, r_{1}, v_{1}, \ldots, r_{n}$, $v_{n}=v^{\prime}$ такая, что для любого $i=1,2, \ldots, n$ справедливы равенства $v_{i-1}=l^{-}\left(r_{i}\right)$, $v_{i}=l^{+}\left(r_{i}\right)$ или $v_{i-1}=l^{+}\left(r_{i}\right), v_{i}=l^{-}\left(r_{i}\right)$.

Согласно кластерной теореме [10] имеем

$$
U=: e^{U_{\mathrm{c}}}:
$$

где индекс "с" указывает, что оставляются только связные графы Фридрихса, а : : означает нормальное упорядочение [10].

Теперь сформулируем основной результат.

Теорема 1. Если $d \geqslant 3$, то справедливо равенство

$$
\left\langle 0\left|e^{-i t H}\right| 0\right\rangle=e^{A t+B+C(t)}
$$

в смысле формальных степенных рядов по $\lambda$. Здесь $A, B, C(t)$ - бормальные степенные ряды по $\lambda$ :

$$
A=\sum_{n=2}^{\infty} A_{n} \lambda^{n}, \quad B=\sum_{n=2}^{\infty} B_{n} \lambda^{n}, \quad C(t)=\sum_{n=2}^{\infty} C_{n}(t) \lambda^{n}
$$

$u C_{n}(t) \rightarrow 0$ npu $t \rightarrow \infty$.

НАЧАЛО ДОКАЗАТЕЛЬСТВА. Имеем

$$
\left\langle 0\left|e^{-i t H}\right| 0\right\rangle=\left\langle 0\left|e^{i t H_{0}} e^{-i t H}\right| 0\right\rangle=e^{\left\langle 0\left|U_{\mathrm{c}}(t)\right| 0\right\rangle},
$$

где

$$
\left\langle 0\left|U_{\mathrm{c}}(t)\right| 0\right\rangle=\sum_{n=1}^{\infty}(-i \lambda)^{n+1} F_{n}(t),
$$

а $F_{n}(t)$ - сумма по всем связным графам Фридрихса порядка $n+1$ выражений вида

$$
F_{n}^{\Phi}(t)=\int_{0}^{t} d t_{n} \cdots \int_{0}^{t_{1}} d t_{0} \int e^{i\left(E_{n} t_{n}+\cdots+E_{0} t_{0}\right)} f(p) d p .
$$

Здесь $f(p)$ - произведение ядер мономов,

$$
E_{i}=\sum_{l^{-}(r)=v} \omega\left(p_{r}\right)-\sum_{l^{+}(r)=v} \omega\left(q_{r}\right)
$$

$\left\{p_{r}\right\}$ - импульсы, соответствующие ребрам $\left\{r \mid l^{-}(r)=v\right\}$, a $\left\{q_{r}\right\}-$ импульсы, соответствующие ребрам $\left\{r \mid l^{+}(r)=v\right\}$. 
Преобразуем $F_{n}^{\Phi}$. Имеем

$$
\begin{aligned}
F_{n}^{\Phi}(t) & =\int_{0}^{t} d t_{n} \int_{0}^{t_{n}} d t_{n-1} \ldots \int_{0}^{t_{1}} d t_{0} \int e^{i\left(E_{n} t_{n}+\cdots+E_{0} t_{0}\right)} f(p) d p= \\
& =\int_{0}^{t} d t_{0} \int_{0}^{t-t_{0}} d t_{n} \ldots \int_{0}^{t_{2}} d t_{1} \int e^{i\left(E_{n} t_{n}+\cdots+E_{1} t_{1}\right)} f(p) d p= \\
& =\int_{0}^{t} d t_{0} \int_{0}^{t_{0}} d t_{n} \ldots \int_{0}^{t_{2}} d t_{1} \int e^{i\left(E_{n} t_{n}+\cdots+E_{1} t_{1}\right)} f(p) d p .
\end{aligned}
$$

Возьмем $T>t$. Тогда

$$
\begin{aligned}
F_{n}^{\Phi}(t)= & \int_{0}^{t} d t_{0} \int_{0}^{T} d t_{n} \ldots \int_{0}^{t_{2}} d t_{1} \int e^{i\left(E_{n} t_{n}+\cdots+E_{1} t_{1}\right)} f(p) d p- \\
& -\int_{0}^{t} d t_{0} \int_{t_{0}}^{T} d t_{n} \cdots \int_{0}^{t_{2}} d t_{1} \int e^{i\left(E_{n} t_{n}+\cdots+E_{1} t_{1}\right)} f(p) d p .
\end{aligned}
$$

Функция

$$
\int e^{i\left(E_{n} t_{n}+\cdots+E_{1} t_{1}\right)} f(p) d p
$$

есть непрерывная функция и потому локально интегрируема. Заметим, что теорема будет доказана, если мы покажем, что следующие пределы существуют:

$$
\begin{aligned}
& A_{n}^{\Phi}=\lim _{T \rightarrow \infty} \int_{0}^{T} d t_{n} \ldots \int_{0}^{t_{2}} d t_{1} \int e^{i\left(E_{n} t_{n}+\cdots+E_{1} t_{1}\right)} f(p) d p \\
& B_{n}^{\Phi}=-\lim _{t \rightarrow \infty} \lim _{T \rightarrow \infty} \int_{0}^{t} d t_{0} \int_{t_{0}}^{T} d t_{n} \ldots \int_{0}^{t_{2}} d t_{1} \int e^{i\left(E_{n} t_{n}+\cdots+E_{1} t_{1}\right)} f(p) d p .
\end{aligned}
$$

Обозначим через $A_{n}$ сумму выражений (1), взятую по всем графам степени $n$, а через $B_{n}$ - сумму выражений (2). Получим

$$
F_{n}(t)=A_{n} t+B_{n}+C_{n}(t)
$$

где $C_{n}(t) \rightarrow 0$ при $t \rightarrow \infty$. Ниже мы докажем, что пределы $(1),(2)$ существуют, если $d \geqslant 3$.

\section{3. СХОДИМОСТЬ ВЫРАЖЕНИЙ ДЛЯ $A_{n}$}

Зафиксируем граф Фридрихса $\Phi$. Получим

$$
I:=A_{n}^{\Phi}=\int_{0}^{\infty} d t_{n} \cdots \int_{0}^{t_{2}} d t_{1} \int e^{i\left(E_{n} t_{n}+\cdots+E_{1} t_{1}\right)} f(p) d p .
$$

Граф Фридрихса $\Phi=\left(V, R, l^{+}, l^{-}\right)$, фигурирующий в $(3)$, связный.

Напомним, что через $V$ мы обозначили множество всех вершин, за исключением минимальной. Произвольный элемент из $V$ мы обозначим через $v, v \in V$. Множество $V$ мы можем отождествить с множеством $\{1,2, \ldots, n\}$. 
Для любого $r \in R$ определим множество $V_{r}$ :

$$
V_{r}:=\left\{v \mid l^{+}(r) \geqslant v>v-1 \geqslant l^{-}(r)\right\} .
$$

Для любого $v \in V$ определим множество $R_{v}$ :

$$
R_{v}:=\left\{r \mid l^{+}(r) \geqslant v>v-1 \geqslant l^{-}(r)\right\} .
$$

Очевидно, что $v \in V_{r} \Leftrightarrow r \in R_{v}$. Для любого $A \subset V$ положим

$$
R_{A}=\left\{r \mid \exists v \in A: r \in R_{v}\right\} .
$$

Введем новые переменные интегрирования $\tau_{i}:=t_{i}-t_{i-1}, i=1,2, \ldots, n$. Получим

$$
\begin{aligned}
I=\int_{0}^{\infty} d \tau_{n} \ldots \int_{0}^{\infty} d \tau_{1} \exp \left(i \omega_{0} \sum_{r \in R} \sum_{v \in V_{r}} \tau_{v}\right) \times \\
\quad \times \int \prod_{r} d p_{r} \exp \left(\frac{i}{2} \sum_{r \in R} p_{r}^{2} \sum_{v \in V_{r}} \tau_{v}\right) f\left(\ldots p_{r} \ldots\right) .
\end{aligned}
$$

Сначала выберем

$$
f\left(\ldots p_{r} \ldots\right)=\exp \left(-\frac{1}{2} \sum_{r \in R} p_{r}^{2}\right)
$$

Тогда интеграл по импульсам $p_{r}$ берется явно, получим

$$
I=\int_{0}^{\infty} d \tau_{n} \ldots \int_{0}^{\infty} d \tau_{1} \exp \left(i \omega_{0} \sum_{r \in R} \sum_{v \in V_{r}} \tau_{v}\right) \prod_{r}\left\{(2 \pi)^{d / 2}\left(1+i \sum_{v \in V_{r}} \tau_{v}\right)^{-d / 2}\right\} .
$$

Наша цель - выяснить, когда интеграл $I$ существует.

Пусть $A \subset V$ и $\mathcal{O}_{A}$ подмножество в $\mathbb{R}_{+}^{n}$, определенное следующим образом:

$$
\mathcal{O}_{A}=\left\{\left(\tau_{1}, \ldots, \tau_{n}\right) \mid \tau_{i}<1 \text {, если } i \in A ; \tau_{i} \geqslant 1 \text {, если } i \notin A\right\} .
$$

Интеграл $I$ сходится тогда и только тогда, когда он сходится по каждой из областей $\mathcal{O}_{A}$. Интеграл $I$ по множеству $\mathcal{O}_{\varnothing}$ существует, если существует интеграл

$$
J=\int_{1}^{\infty} d \tau_{n} \ldots \int_{1}^{\infty} d \tau_{1} \prod_{r}\left(\sum_{v \in V_{r}} \tau_{v}\right)^{-d / 2} .
$$

Для оценки этого интеграла используем неравенство Гельдера

$$
\left|\int d \tau \prod_{k=1}^{n} f_{k}(\tau)\right| \leqslant \prod_{k=1}^{n}\left(\int d \tau\left|f_{k}(\tau)\right|^{q_{k}}\right)^{1 / q_{k}}, \quad 0<q_{k} \leqslant \infty, \quad \sum_{k=1}^{n} \frac{1}{q_{k}}=1 .
$$

Существование левой части этого неравенства следует из существования правой. Интегрируя по $\tau_{1}$, получаем

$$
J \leqslant C_{1} \int_{1}^{\infty} d \tau_{n} \ldots \int_{1}^{\infty} d \tau_{2} \prod_{r}\left(\sum_{v \in V_{r}, v \neq 1} \tau_{v}\right)^{-\left(d / 2-\left(q_{r}^{1}\right)^{-1}\right)} .
$$


По определению $q_{r}^{1}$ выбраны так, что $q_{r}^{1}=\infty$, если $r \notin R_{1}$ и $\sum_{r \in R} 1 / q_{r}=1$.

Интеграл по $\tau_{1}$ существует, если существует набор коэффициентов $q_{r}^{1}$ такой, что $d / 2-\left(q_{r}^{1}\right)^{-1}>0$.

Проинтегрируем теперь по $\tau_{2}$. Получим

$$
J \leqslant C_{2} \int_{1}^{\infty} d \tau_{n} \ldots \int_{1}^{\infty} d \tau_{3} \prod_{r}\left(\sum_{v \in V_{r}, v \neq 1,2} \tau_{v}\right)^{-\left(d / 2-\left(q_{r}^{1}\right)^{-1}-\left(q_{r}^{2}\right)^{-1}\right)} .
$$

Интеграл по $\tau_{2}$ существует, если $d / 2-\left(q_{r}^{1}\right)^{-1}-\left(q_{r}^{2}\right)^{-1}>0$. Заметим, что отсюда следует, что $d / 2-\left(q_{r}^{1}\right)^{-1}>0$. По индукции можно показать, что $J$ существует, если выполнено следующее условие.

А. Найдутся такие действительные числа $0 \leqslant s_{r}^{v}<\infty$, что

$$
\sum_{r \in R_{v}} s_{r}^{v}=1, \quad \sum_{v \in V_{r}} s_{r}^{v}<\frac{d}{2} .
$$

Это типичная задача линейного программирования (см. [21]). Условие А эквивалентно следующему условию.

Б. Найдутся такие действительные числа $0 \leqslant s_{r}^{v}<\infty$, что

$$
\begin{aligned}
& \sum_{r \in R_{v}} s_{r}^{v}>1, \\
& \sum_{v \in V_{r}} s_{r}^{v}=\frac{d}{2} .
\end{aligned}
$$

Докажем, что из условия Б следует условие А. Уменьшая все ненулевые $s_{r}^{v}$ на достаточно малые ненулевые величины, мы сохраним неравенство (7), а в (8) получим строгое неравенство. Затем, перебирая все вершины, начиная с $v=1$ и заканчивая $v=n$, уменьшая $s_{r}^{v}$, мы добьемся того, чтобы (7) превратилось в равенство.

Введем понятие фактор-графа. Фактор-граф $\Phi_{A}, A \subset V$, является результатом последовательного действия операций $T_{i}$. По определению $T_{i}$ - граф, множество вершин которого есть $T_{i}:=V-\{i\}$, а множество ребер $T_{i} R$ состоит из всех ребер, для которых либо $l^{+}(r) \neq i$, либо $l^{-}(r) \neq i-1$. Функции $l^{+}, l^{-}$для графа $T_{i} \Phi$ суть ограничения соответствующих функций для графа $\Phi$ на $T_{i} R$.

Положим по определению

$$
\Phi_{A}=\prod_{i \in V \backslash A} T_{i} \Phi,
$$

причем сомножители располагаются в порядке возрастания номеров слева направо.

Степенью расходимости графа $\Phi$ называется величина

$$
C_{\Phi}=|V|-\frac{d}{2}|R|
$$

Наша задача будет состоять в том, чтобы найти условия, необходимые и достаточные для выполнения условия Б. Если выполнено условие Б, то индекс расходимости 
графа и всех его фактор-графов отрицателен. Проверим это только для самого графа:

$$
\frac{d}{2}|R|=\sum_{r \in R} \sum_{v \in V_{r}} s_{r}^{v}=\sum_{v \in V} \sum_{r \in R_{v}} s_{r}^{v}>\sum_{v \in V_{r}} 1=|V| .
$$

Итак,

$$
|V|-\frac{d}{2}|R|<0
$$

Верно и обратное.

УтВеРжДЕНИЕ. Если индекс расходимости графа и всех его фактор-графов отрицателен, то выполнено условие Б.

ДокАЗАТЕЛЬСтво. Обозначим набор коэффициентов $s_{r}^{v}$, удовлетворяющих условию (8) и условию $s_{r}^{v}=0$, если $r \notin R_{v}$, буквой $C$, а множество таких наборов символом С. Множество $\mathbf{C}$ можно отождествить с подмножеством в $\mathbb{R}^{N}$, где $N=$ $\sum_{v \in V_{r}} \sum_{r \in R_{v}}$ 1. Это множество выпуклое. Последнее означает, что наряду с $C_{1} \in \mathbf{C}$ и $C_{2} \in \mathbf{C}$ имеет место $m_{1} C_{1}+m_{2} C_{2} \in \mathbf{C}$, где $m_{1} \geqslant 0, m_{2} \geqslant 0, m_{1}+m_{2}=1$.

Введем следующие обозначения:

$$
C_{v}:=\sum_{r \in R_{v}} s_{r}^{v}, \quad|C|:=\sum_{v \in V} C_{v}=\frac{d}{2}, \quad C_{A}:=\sum_{v \in A} C_{v} .
$$

Предположим, что удалось подобрать такие $C^{k}, k=1,2, \ldots, n$, что $C_{v}^{k}>1$, если $k \neq v$.

Рассмотрим $C_{1}^{1}$. Если $C_{1}^{1}>1$, то утверждение доказано. Если $C_{1}^{1} \leqslant 1$, то для любого произвольно малого $\varepsilon$ рассмотрим выпуклую линейную комбинацию $m_{1} C^{1}+$ $m_{2} C^{2}$ такую, что

$$
\begin{aligned}
\left|m_{1} C^{1}+m_{2} C^{2}\right|_{\{l\}} & >1, \quad l \neq 2, \\
\left|m_{1} C^{1}+m_{2} C^{2}\right|_{V \backslash\{1\}} & >\frac{d}{2}|R|-1-\varepsilon .
\end{aligned}
$$

Таким же образом для любого $k=2,3, \ldots, n$ можно найти элемент $\widetilde{C}^{k}$ такой, что

$$
\begin{aligned}
& \left|\widetilde{C}^{k}\right|_{V \backslash\{1\}}>\frac{d}{2}|R|-1-\varepsilon, \\
& \widetilde{C}_{l}^{k}>1, \quad \text { если } \quad k \neq l .
\end{aligned}
$$

Если найдется $k$ такое, что $\widetilde{C}_{k}^{k}>1$, утверждение доказано. В противном случае можно построить наборы $\widetilde{\widetilde{C}}^{k}, k=3,4, \ldots, n$, такие, что $\widetilde{\widetilde{C}}_{l}^{k}>1$, если $k \neq l$ и

$$
|\widetilde{\widetilde{C}}|_{V \backslash\{1,2\}}^{k}>\frac{d}{2}|R|-2-\varepsilon .
$$

Эта индуктивная процедура либо благополучно прервется, либо мы построим набор $\widehat{C}$ такой, что

$$
\widehat{C}_{k}>1, \quad k \neq n,
$$




$$
\widehat{C}_{n}>\frac{d}{2}|R|-V+1-\varepsilon>1,
$$

если $\varepsilon$ достаточно мало.

Итак, необходимо построить наборы $C^{k}$. Тем же способом, что и выше, найдем, что достаточно построить наборы $C^{k, l}$ такие, что $C_{v}^{k, l}>1$, если $v \neq k, l,\left|C^{k, l}\right| \geqslant$ $(d / 2)|R|$ (автоматически выполнено) и $\left|C^{k, l}\right|_{V \backslash\{k\}}>(d / 2)|R|_{V \backslash\{k\}}$. Кроме того, так же, как выше, найдем, что для построения $C^{k, l}$ достаточно построить наборы $C^{k, l, m}$ такие, что $C_{v}^{k, l, m}>1$, если $v \neq k, l, m$ и $\left|C^{k, l, m}\right|_{V \backslash\{k\}}>(d / 2)|R|_{V \backslash\{k\}},\left|C^{k, l, m}\right|_{V \backslash\{k, l\}}>$ $(d / 2)|R|_{V \backslash\{k, l\}} \cdot$

Легко видеть, что для завершения доказательства достаточно для каждой перестановки $P$ множества $\{1,2, \ldots, n\}$ построить наборы $C^{P} \in \mathbf{C}$ такие, что

$$
\left|C^{P}\right|_{\{P(1), \ldots, P(k)\}}=\frac{d}{2}|R|_{\{P(1), \ldots, P(k)\}} .
$$

Легко видеть, что это можно сделать по индукции, переходя от фактор-графа $\Phi_{\{P(1), \ldots, P(k)\}}$ к $\Phi_{\{P(1), \ldots, P(k+1)\}}$. В самом деле, $\Phi_{\{P(1), \ldots, P(k)\}}$ есть фактор-граф графа $\Phi_{\{P(1), \ldots, P(k+1)\}}$. Пусть для $\Phi_{\{P(1), \ldots, P(k)\}}$ такой набор коэффициентов уже построен. Построим $s_{r}^{P(k+1)}$ следующим образом:

1) если $r \notin R_{P(k+1)}$, то $s_{r}^{P(k+1)}=0$;

2) если $r \in R_{P(k+1)}$ и $r \in R_{\{P(1), \ldots, P(k)\}}$, то $s^{P}(k+1)_{r}=0$;

3) если $r \in R_{P(k+1)}$ и $r \notin R_{\{P(1), \ldots, P(k)\}}$, то $s_{r}^{P(k+1)}=d / 2$. Тогда

$$
\begin{aligned}
& \sum_{v \in\{P(1), \ldots, P(k+1)\}} \sum_{r \in R_{v}} s_{r}^{v}=\sum_{v \in\{P(1), \ldots, P(k)\}} \sum_{r \in R_{v}} s_{r}^{v}+\sum_{r \in R_{P(k+1)}} s_{r}^{P(k+1)}= \\
& =\frac{d}{2}\left|R_{\{P(1), \ldots, P(k)\}}\right|+\left(\frac{d}{2}\left|R_{\{P(1), \ldots, P(k+1)\}}\right|-\frac{d}{2}\left|R_{\{P(1), \ldots, P(k)\}}\right|\right)= \\
& =\frac{d}{2}\left|R_{\{P(1), \ldots, P(k+1)\}}\right| .
\end{aligned}
$$

Итак, доказано, что для сходимости интеграла $I$ по области $O_{\varnothing}$ достаточно, чтобы индекс расходимости $\Phi$ и всех его фактор-графов был отрицательным. Анализ сходимости $I$ по другим областям $O_{A}, A \neq \varnothing$, аналогичен только что проведенному. Он сводится к анализу сходимости интеграла $J$ для $\Phi_{V \backslash A}$. Итак, доказана

Теорема 2. Для абсолютной сходимости интеграла I (5) достаточно, чтобы индекс расходимости графа и всех его фактор-графов был отрицательным.

Теорема 3. Если $d \geqslant 3$, то выполнены все условия теоремы 2 и интеграл I (5) сходится абсолютно.

ДокАЗАТЕЛЬСтво. Покажем, что для произвольного связного графа Фридрихса $|R| \geqslant|V|$. Для вершины с номером 0 найдутся вершина с номером $i_{1}$ и ребро, их соединяющее, в противном случае вершина с номером 0 есть нетривиальная компонента связности графа $\Phi$. Для вершин с номерами $0, i_{1}$ найдутся вершина с 
номером $i_{2}$ и ребро, которое соединяет ее с вершиной с номером 0 или $i_{1}$, в противном случае вершины $0, i_{1}$ и ребра, их соединяющие, образуют нетривиальную компоненту связности, и т.д. Итак, ребер, по крайней мере, не меньше, чем число вершин минус 1.

Теорема 4. Интеграл I (4) сходится для произволъной функции $f(p)$ из пространства Швариа.

ДокАЗАтЕЛьство. Интеграл (4) мы, как и в доказательстве теоремы 2, представим в виде $I=\sum_{V \subset A} I_{A}$, где $I_{A}$ имеет ту же подынтегральную функцию, что и $I$, но берется по области $\mathcal{O}_{A}$. Оценим подынтегральную функцию в $\mathcal{O}_{A}$. Пусть $F$ означает преобразование Фурье по переменным $p_{r}, r \in R_{B}, B=V \backslash A$ (о преобразовании Фурье для обобщенных функций см. [22]). Тогда имеем

$$
\begin{aligned}
\left|\int \prod_{r} d p_{r} \exp \left(\frac{i}{2} \sum_{r \in R} p_{r}^{2} \sum_{v \in V_{r}} \tau_{v}\right) F\left(F^{-1}(f)\right)\left(\ldots p_{r} \ldots\right)\right|= \\
=\mid \int \prod_{r} d p_{r} F\left(\exp \left(\frac{i}{2} \sum_{r \in R_{B}} p_{r}^{2} \sum_{v \in V_{r}} \tau_{v}\right)\right) \times \\
\quad \times \exp \left(\frac{i}{2} \sum_{r \notin R_{B}} p_{r}^{2} \sum_{v \in V_{r}} \tau_{v}\right)\left(F^{-1}\left(f\left(\ldots p_{r} \ldots\right)\right)\right) \mid= \\
=\mid \prod_{r \in R_{B}}(2 \pi)^{d / 2}\left(\sum_{v \in V_{r}} \tau_{v}\right)^{-1} \times \\
\quad \times \int \prod_{r} d p_{r}\left(\exp \left(-\frac{i}{2} \frac{\sum_{r \in R_{B}} p_{r}^{2}}{\sum_{v \in V_{r}} \tau_{v}}\right)\right) \times \\
\quad \times \exp \left(-\frac{i}{2} \sum_{r \notin R_{B}} p_{r}^{2} \sum_{v \in V_{r}} \tau_{v}\right)\left(F^{-1}\left(f\left(\ldots p_{r} \ldots\right)\right)\right) \mid \leqslant \\
\leqslant \operatorname{const} \cdot \prod_{r \in R_{B}}\left(\sum_{v \in V_{r}^{B}} \tau_{v}\right)^{-1},
\end{aligned}
$$

где $V_{r}^{B}:=V_{r} \cap B$. Именно эта оценка использовалась для доказательства теоремы 2.

\section{4. КОЭФФИЦИЕНТ $B$}

Фиксируем некоторый граф Фридрихса $\Phi$. Тогда

$$
\begin{aligned}
L:= & B_{n}^{\Phi}=\lim _{t \rightarrow \infty} \lim _{T \rightarrow \infty} \int_{0}^{t} d t_{0} \int_{t_{0}}^{T} d t_{n} \ldots \int_{0}^{t_{2}} d t_{1} \int e^{i\left(E_{n} t_{n}+\cdots+E_{1} t_{1}\right)} f(p) d p= \\
= & \lim _{t \rightarrow \infty} \lim _{T \rightarrow \infty} \int_{\tau_{1}+\cdots+\tau_{n}<T ; \tau_{i}>0} \prod_{i=1}^{n} d \tau^{i} \min \left(t, \tau_{1}+\cdots+\tau_{n}\right) \times \\
& \times \exp \left(i \omega_{0} \sum_{r \in R} \sum_{v \in V_{r}} \tau_{v}\right) \int \prod_{r} d p_{r} \exp \left(\frac{i}{2} \sum_{r \in R} p_{r}^{2} \sum_{v \in V_{r}} \tau_{v}\right) f\left(\ldots p_{r} \ldots\right) .
\end{aligned}
$$


Наша задача состоит в том, чтобы доказать, что при выполнении условий теоремы 2 этот интеграл сходится. Представим его в виде суммы $L=\sum_{A \subset V} L_{A}$, где $L_{A}$ имеет ту же подынтегральную функцию, что и $L$, но берется по области $\mathcal{O}_{A}$. Для простоты проведем анализ только для $L_{\varnothing}$. Сначала в формуле для $L_{\varnothing}$ поменяем порядок вычисления пределов, докажем сходимость полученного выражения, а затем обоснуем законность такой процедуры. Поменяв порядок вычисления пределов, получим интеграл

$$
\begin{aligned}
K_{\varnothing}(T)= & \int_{\tau_{1}+\cdots+\tau_{n}<T ; \tau_{i}>1} \prod_{i=1}^{n} d \tau^{i}\left(\tau_{1}+\cdots+\tau_{n}\right) \times \\
& \times \exp \left(i \omega_{0} \sum_{r \in R} \sum_{v \in V_{r}} \tau_{v}\right) \int \prod_{r} d p_{r} \exp \left(\frac{i}{2} \sum_{r \in R} p_{r}^{2} \sum_{v \in V_{r}} \tau_{v}\right) f\left(\ldots p_{r} \ldots\right),
\end{aligned}
$$

или

$$
\begin{aligned}
K_{\varnothing}(T)= & \int_{\tau_{1}+\cdots+\tau_{n}<T ; \tau_{i}>1} \prod_{i=1}^{n} d \tau^{i}\left(\tau_{1}+\cdots+\tau_{n}\right) \exp \left(i \omega_{0} \sum_{r \in R} \sum_{v \in V_{r}} \tau_{v}\right) \times \\
& \times \prod_{r} \frac{1}{(2 \pi)^{d / 2}}\left(\sum_{v \in V_{r}} \tau_{v}\right)^{-d / 2} f\left(\tau_{1}, \ldots, \tau_{n}\right),
\end{aligned}
$$

где

$$
f\left(\tau_{1}, \ldots, \tau_{n}\right)=(-i)^{d|R| / 2} \int \prod_{r \in R} d x_{r} F^{-1}(f)\left(x_{r}\right) \exp \left(-\frac{i}{2} \sum_{r \in R} x_{r}^{2}\left(\sum_{v \in V_{r}} \tau_{v}\right)^{-1}\right) .
$$

Лемма 1. Справедлива следующая оценка:

$$
\left|\frac{d}{d \tau} f\left(\tau, \ldots, \tau, \tau_{k+1}, \ldots, \tau_{n}\right)\right| \leqslant \frac{\text { const }}{\tau} .
$$

ДокАЗАТЕЛьство. Имеем

$$
\begin{gathered}
\frac{d}{d \tau} f\left(\tau, \ldots, \tau, \tau_{k+1}, \ldots, \tau_{n}\right)=\frac{1}{2} \prod_{r \in R} \int d x_{r} F^{-1}(f)\left(x_{r}\right) \exp \left(-\frac{i}{2} \sum_{r \in R} x_{r}^{2}\left(\sum_{v \in V_{r}} \tau_{v}\right)^{-1}\right) \times \\
\times\left.\sum_{v \in\{1, \ldots, k\}} \sum_{r \in R_{v}} x_{r}^{2}\left(\sum_{v \in V_{r}} \tau_{v}\right)^{-2}\right|_{\tau_{1}=\cdots=\tau_{k}=\tau} .
\end{gathered}
$$

Но $\forall v \in\{1,2, \ldots, k\}$ справедливо неравенство

$$
\left.\sum_{r \in R_{v}}\left(\sum_{v \in V_{r}} \tau_{v}\right)^{-2}\right|_{\tau_{1}=\cdots=\tau_{k}=\tau} \leqslant \frac{\text { const }}{\tau} .
$$

Мы можем оценить интеграл в (11) сверху константой. Лемма доказана. 
ЛЕмма 2. В условиях теоремы 2 сходится интеграл

$$
\begin{aligned}
\lim _{T \rightarrow \infty} K_{\varnothing}(T)^{k}=\lim _{T \rightarrow \infty} \int_{D_{k, n}} \prod_{i=k}^{n} d \tau_{i}\left(\tau_{1}+\cdots+\tau_{n}\right) \exp \left(i \omega_{0} \sum_{r \in R} \sum_{v \in V_{r}} \tau_{v}\right) \times \\
\times\left.\prod_{r \in R} \frac{1}{(2 \pi)^{d / 2}}\left(\sum_{v \in V_{r}} \tau_{v}\right)^{-d / 2} f\left(\tau_{1}, \ldots, \tau_{n}\right)\right|_{\tau_{1}=\cdots=\tau_{k}},
\end{aligned}
$$

где $D_{k, n}=\left\{k \tau_{k}+\cdots+\tau_{n}<T ; \tau_{i}>1 ; \tau_{k}>\cdots>\tau_{n}\right\}$.

ДоказАтельство. Сначала рассмотрим случай $k=n$. Интеграл в (12) равен

$$
\begin{aligned}
K_{\varnothing}^{n}(T)=n & \int_{1}^{T / n} d \tau \tau \exp \left(i \omega_{0} \sum_{r \in R}\left|V_{r}\right| \tau\right) \prod_{r \in R} \frac{1}{(2 \pi)^{d / 2}}\left(\sum_{v \in V_{r}} \tau\right)^{-d / 2} f(\tau, \ldots, \tau)= \\
= & \frac{n}{i \omega_{0} N}\left\{\left.\frac{T}{n} \exp \left(i \omega_{0} \sum_{r \in R}\left|V_{r}\right| \frac{T}{n}\right) \prod_{r \in R} \frac{1}{(2 \pi)^{d / 2}}\left(\sum_{v \in V_{r}} \tau\right)^{-d / 2} f(\tau, \ldots, \tau)\right|_{\tau=T / n}-\right. \\
& -\exp \left(i \omega_{0} \sum_{r \in R}\left|V_{r}\right|\right) \prod_{r \in R} \frac{1}{(2 \pi)^{d / 2}} \frac{1}{\left(\left|V_{r}\right|\right)^{d / 2}} f(1, \ldots, 1)+ \\
& +\left(\frac{d}{2}|R|-1\right) \int_{1}^{T / n} d \tau \frac{1}{\tau^{(d / 2)|R|}} \prod_{r \in R} \frac{1}{(2 \pi)^{d / 2}} \frac{1}{\left(\left|V_{r}\right|\right)^{d / 2}} \times \\
& \times \exp \left(i \omega_{0} \sum_{r \in R}\left|V_{r}\right| \tau\right) f(\tau, \ldots, \tau)- \\
& -\prod_{r \in R} \frac{1}{(2 \pi)^{d / 2}} \frac{1}{\left(\left|V_{r}\right|\right)^{d / 2}} \int_{1}^{T / n} d \tau \frac{\tau}{\tau^{(d / 2)|R|}} \frac{d}{d \tau} f(\tau, \ldots, \tau) \times \\
& \left.\times \exp \left(i \omega_{0} \sum_{r \in R}\left|V_{r}\right| \tau\right)\right\} .
\end{aligned}
$$

Напомним,что $(d / 2)|R|>|V| \geqslant 1$. Следовательно, первое слагаемое стремится к нулю, второе постоянно, интеграл в третьем слагаемом сходится, а интеграл в четвертом слагаемом сходится в силу того, что

$$
\left|\frac{d}{d \tau} f(\tau, \ldots, \tau)\right| \leqslant \frac{\text { const }}{\tau} .
$$

Допустим, что предположение индукции справедливо при $k+1$, докажем его справедливость при $k$. Интегрируя по частям по $d \tau_{k}$, получаем

$$
\begin{aligned}
K_{\varnothing}(T)^{k}= & \int_{D_{k, n}}\left(\tau_{1}+\cdots+\tau_{n}\right) \exp \left(i \omega_{0} \sum_{r \in R} \sum_{v \in V_{r}} \tau_{v}\right) \times \\
& \times\left.\prod_{r} \frac{1}{(2 \pi)^{d / 2}}\left(\sum_{v \in V_{r}} \tau_{v}\right)^{-d / 2} f\left(\tau_{1}, \ldots, \tau_{n}\right)\right|_{\tau_{1}=\cdots=\tau_{k}} \prod_{i=k}^{n} d \tau_{i}= \\
= & \frac{1}{i \omega_{0} N}\left\{T \int_{\substack{(k+1) \tau_{k+1}+\cdots+\tau_{n}<T \\
\tau_{i}>1 ; \tau_{k+1}>\cdots>\tau_{n}}} \prod_{i=k+1}^{n} d \tau_{i} \prod_{r \in R} \frac{1}{(2 \pi)^{d / 2}}\left(\sum_{v \in V_{r}} \tau_{v}\right)^{-d / 2} \times\right.
\end{aligned}
$$




$$
\begin{aligned}
& \times\left.\exp \left(i \omega_{0} \sum_{r \in R} \sum_{v \in V_{r}} \tau_{v}\right) f\left(\tau_{1}, \ldots, \tau_{n}\right)\right|_{\tau_{1}=\cdots=\tau_{k}=\left(T-\tau_{k+1}-\cdots-\tau_{n}\right) / k}- \\
& -\int_{\substack{(k+1) \tau_{k+1}+\cdots+\tau_{n}<T ; \\
\tau_{i}>1 ; \tau_{k+1}>\cdots>\tau_{n}}} \prod_{i=k+1}^{n} d \tau_{i} \prod_{r \in R} \frac{1}{(2 \pi)^{d / 2}}\left(\sum_{v \in V_{r}} \tau_{v}\right)^{-d / 2} \times \\
& \times\left.\exp \left(i \omega_{0} \sum_{r \in R} \sum_{v \in V_{r}} \tau_{v}\right)\left(\tau_{1}+\cdots+\tau_{n}\right) f\left(\tau_{1}, \ldots, \tau_{n}\right)\right|_{\tau_{1}=\cdots=\tau_{k}=\tau_{k+1}}- \\
& -\int_{D_{k, n}} \prod_{i=k}^{n} d \tau_{i} \frac{d}{d \tau_{k}}\left(\left\{\left(k \tau_{k}+\cdots+\tau_{n}\right) f\left(\tau_{1}, \ldots, \tau_{n}\right) \times\right.\right. \\
& \left.\times \prod_{r} \frac{1}{(2 \pi)^{d / 2}}\left(\sum_{v \in V_{r}} \tau_{v}\right)^{-d / 2}\right\}\left.\right|_{\tau_{1}=\cdots=\tau_{k}} \times \exp \left(i \omega_{0} \sum_{r \in R} \sum_{v \in V_{r}} \tau_{v}\right)
\end{aligned}
$$

где $N=\sum_{l=1}^{k} \sum_{r \in R_{l}} 1$. Второе слагаемое есть $K_{\varnothing}^{k+1}(T)$ и потому сходится при $T \rightarrow \infty$. Первое слагаемое будем оценивать с помощью неравенства Гельдера с коэффициентами, выбранными при доказательстве теоремы 2. Заметим, что $\tau_{1}=$ $\cdots=\tau_{k}>T / n$. Обозначим первое слагаемое через $Q$. Тогда

$$
|Q| \leqslant T \cdot \text { const } \prod_{r \in R_{\{1, \ldots, k\}}} T^{-\left[\frac{d}{2}-\sum_{v=k+1}^{n}\left(q_{r}^{k}\right)^{-1}\right]} .
$$

Но в силу выбора $\left(q_{r}^{k}\right)$ имеем

$$
\frac{d}{2}-\sum_{v=k+1}^{n}\left(q_{r}^{k}\right)^{-1} \geqslant\left(q_{r}^{1}\right)^{-1}-\varepsilon
$$

для некоторого $\varepsilon$. Поэтому

$$
|Q| \leqslant \frac{1}{T^{\varepsilon}} \frac{T}{T^{\sum_{r}\left(q_{r}^{1}\right)^{-1}}}=\frac{1}{T^{\varepsilon}} \rightarrow 0,
$$

так первое слагаемое стремится к нулю. Третье слагаемое раскроем по правилу Лейбница. Получим сумму трех слагаемых:

$$
\begin{aligned}
&\left.k \int_{D_{k, n},} \prod_{i=k}^{n} d \tau_{i} \exp \left(i \omega_{0} \sum_{r \in R} \sum_{v \in V_{r}} \tau_{v}\right) \prod_{r \in R} \frac{1}{(2 \pi)^{d / 2}}\left(\sum_{v \in V_{r}} \tau_{v}\right)^{-d / 2} f\left(\tau_{1}, \ldots, \tau_{n}\right)\right|_{\tau_{1}=\cdots=\tau_{k}}- \\
&-\frac{d}{2} \int_{D_{k, n}} \prod_{i=k}^{n} d \tau_{i}\left\{\sum_{v \in\{1, \ldots, k\}} \sum_{r \in R_{v}}\left(\sum_{v \in V_{r}} \tau_{v}\right)^{-1}\right\} \times \\
& \times\left(k \tau_{k}+\cdots+\tau_{n}\right) \exp \left(i \omega_{0} \sum_{r \in R} \sum_{v \in V_{r}} \tau_{v}\right) \times \\
& \times\left.\prod_{r \in R} \frac{1}{(2 \pi)^{d / 2}}\left(\sum_{v \in V_{r}} \tau_{v}\right)^{-d / 2} f\left(\tau_{1}+\cdots+\tau_{n}\right)\right|_{\tau_{1}=\cdots=\tau_{k}}+ \\
&+\int_{D_{k, n}} \prod_{i=k}^{n} d \tau_{i}\left(\tau_{1}+\cdots+\tau_{n}\right) \exp \left(i \omega_{0} \sum_{r \in R} \sum_{v \in V_{r}} \tau_{v}\right) \times
\end{aligned}
$$




$$
\times\left.\prod_{r \in R}\left(\sum_{v \in V_{r}} \tau_{v}\right)^{-d / 2} \frac{d}{d \tau_{k}} f\left(\tau_{k}, \ldots, \tau_{k}, \ldots, \tau_{n}\right)\right|_{\tau_{1}=\ldots=\tau_{k}} .
$$

В силу того, что $k \tau_{k}+\cdots+\tau_{n}<\tau_{k} n$ и

$$
\prod_{r}\left(\sum_{v \in V_{r}} \tau_{v}\right)^{-d / 2} \leqslant \prod_{r \in R_{\{k, \ldots, n\}}}\left(\sum_{v \in V_{r} \cap\{k, \ldots, n\}} \tau_{v}\right)^{-d / 2},
$$

разность Коши для члена в последней формуле оценивается сверху разностью Коши для $J$, построенного по фактор-графу $\Phi_{\{k, \ldots, n\}}$, и, следовательно, третье слагаемое в (13) также сходится. Итак, из справедливости леммы при $k+1$ вытекает ее справедливость при $k$. Лемма доказана.

Заметим, что интеграл $K_{\varnothing}$ разбиением области интегрирования разбивается в сумму слагаемых, подобных $K_{\varnothing}(T)^{k}$. Следовательно доказана

Лемма 3. Интеграл $K_{\varnothing}(T)$ сходится при $T \rightarrow \infty$.

Лемма 4. В формуле (9) можно менять местами операчии перехода к пределу.

ДокАЗАТЕЛЬСтво. Перейдя к пределу в $(9)$ по $T \rightarrow \infty$ и вычитая из полученного выражения $K_{\varnothing}(T)$, получим

$$
\begin{aligned}
L_{\varnothing}(t)-K_{\varnothing}(t)=t & \int_{\tau_{1}+\cdots+\tau_{n}>t ; \tau_{i}>1}\left(\tau_{1}+\cdots+\tau_{n}\right) \exp \left(i \omega_{0} \sum_{r \in R} \sum_{v \in V_{r}} \tau_{v}\right) \times \\
& \times \prod_{r} \frac{1}{(2 \pi)^{d / 2}}\left(\sum_{v \in V_{r}} \tau_{v}\right)^{-1} f\left(\tau_{1}, \ldots, \tau_{n}\right) .
\end{aligned}
$$

Разобьем область интегрирования на секторы

$$
\mathcal{O}_{\sigma}=\left\{\left(\tau_{1}, \ldots, \tau_{n}\right) \mid \tau_{i}>1 ; \tau_{\sigma(1)}>\cdots>\tau_{\sigma(n)}\right\},
$$

где $\sigma$ - перестановка элементов множества $\{1,2, \ldots, n\}$. Ограничимся рассмотрением интеграла по $\mathcal{O}_{\mathrm{id}}$. Остальные интегралы рассматриваются аналогично. Проведем интегрирование по частям по $\tau_{1}$. Получим

$$
\begin{aligned}
L_{\varnothing}(T)- & K_{\varnothing}(T)=\frac{T}{i \omega_{0} N}\left\{\lim _{T_{1} \rightarrow \infty} \int_{\substack{T<2 \tau_{2}+\cdots+\tau_{n}<T_{1} ; \\
\tau_{i}>1 ; \tau_{2}>\cdots>\tau_{n}}} \prod_{i=2}^{n} d \tau_{i} \exp \left(i \omega_{0} \sum_{r \in R} \sum_{v \in V_{r}} \tau_{v}\right) \times\right. \\
& \times\left.\prod_{r \in} \frac{1}{(2 \pi)^{d / 2}}\left(\sum_{v \in V_{r}} \tau_{v}\right)^{-d / 2} f\left(\tau_{1}, \ldots, \tau_{n}\right)\right|_{\tau_{1}=T_{1}-\tau_{2}-\cdots-\tau_{n}}- \\
& -\lim _{T_{1} \rightarrow \infty} \int_{\substack{T<2 \tau_{2}+\cdots+\tau_{n}<T_{1} ; \\
\tau_{i}>1 ; \tau_{2}>\cdots>\tau_{n}}} \prod_{i=2}^{n} d \tau_{i} \exp \left(i \omega_{0} \sum_{r \in R} \sum_{v \in V_{r}} \tau_{v}\right) \times \\
& \times\left.\prod_{r \in} \frac{1}{(2 \pi)^{d / 2}}\left(\sum_{v \in V_{r}} \tau_{v}\right)^{-d / 2} f\left(\tau_{1}, \ldots, \tau_{n}\right)\right|_{\tau_{1}=\tau_{2}}-
\end{aligned}
$$




$$
\begin{aligned}
& -\lim _{T_{1} \rightarrow \infty} \int_{\substack{2 \tau_{2}+\cdots+\tau_{n}<T ; \\
\tau_{i}>1 ; \tau_{2}>\cdots>\tau_{n}}} \prod_{i=2}^{n} d \tau_{i} \exp \left(i \omega_{0} \sum_{r \in R} \sum_{v \in V_{r}} \tau_{v}\right) \times \\
& \times\left.\prod_{r \in R} \frac{1}{(2 \pi)^{d / 2}}\left(\sum_{v \in V_{r}} \tau_{v}\right)^{-d / 2} f\left(\tau_{1}, \ldots, \tau_{n}\right)\right|_{\tau_{1}=T-\tau_{2}-\cdots-\tau_{n}}- \\
& -\lim _{T_{1} \rightarrow \infty} \int_{\substack{T<\tau_{1}+\cdots+\tau_{n}<T_{1} ; \\
\tau_{i}>1 ; \tau_{1}>\cdots>\tau_{n}}} \prod_{i=1}^{n} d \tau_{i} \exp \left(i \omega_{0} \sum_{r \in R} \sum_{v \in V_{r}} \tau_{v}\right) \times \\
& \left.\times \frac{d}{d \tau_{1}}\left\{\prod_{r \in R} \frac{1}{(2 \pi)^{d / 2}}\left(\sum_{v \in V_{r}} \tau_{v}\right)^{-d / 2} f\left(\tau_{1}, \ldots, \tau_{n}\right)\right\}\right\}
\end{aligned}
$$

где $N=\sum_{r \in R_{1}} 1$. Первое слагаемое равно нулю. Третье слагаемое стремится к нулю при $T \rightarrow \infty$ (доказательство этого факта такое же, как и для первого слагаемого в (13)). Четвертый член стремится к нулю при $T \rightarrow \infty$ (доказательство аналогично доказательству для четвертого члена в (13)). Что же касается второго члена, то он оценивается так же, как $L_{\varnothing}(t)-K_{\varnothing}(t)$, но в нем уже интегрирований на одно меньше (для оценки $L_{\varnothing}(t)-K_{\varnothing}(t)$ надо выполнить такую же индуктивную процедуру, как и для оценки $\left.K_{\varnothing}(t)\right)$. Лемма доказана.

Объединяя предыдущие четыре леммы, получаем следующую теорему.

Теорема 5. Если выполнены условия теоремы 2, то выражение для $B_{n}$ корректно и представляет собой сумму членов вида

$$
\begin{gathered}
\lim _{T \rightarrow \infty} \int_{\tau_{1}+\cdots+\tau_{n}<T ; \tau_{i}>0}\left(\tau_{1}+\cdots+\tau_{n}\right) \exp \left(i \omega_{0} \sum_{r \in R} \sum_{v \in V_{r}} \tau_{v}\right) \times \\
\times \int \prod_{r} d p_{r} \exp \left(-\frac{i}{2} \sum_{r \in R} p_{r}^{2} \sum_{v \in V_{r}} \tau_{v}\right) f\left(\ldots p_{r} \ldots\right),
\end{gathered}
$$

где $f\left(\ldots p_{r} \ldots\right)$ есть произведение ядер мономов.

Таким образом, мы завершили доказательство теоремы 1.

Заметим, что теорема 2 аналогична теореме о сходимости фейнмановских интегралов в квантовой теории поля.

\section{5. ПРИМЕРЫ}

5.1. Пример к теореме 1. В качестве примера к теореме 1 приведем вычисления во втором порядке по константе связи для гамильтониана взаимодействия

$$
V=\int v\left(p_{1}, \ldots, p_{n}\right) a^{+}\left(p_{1}\right) \ldots a^{+}\left(p_{n}\right)+\text { c.c. }, \quad d \geqslant 3 .
$$

Справедливо равенство

$$
\langle 0|U(t)| 0\rangle=e^{\left\langle 0\left|U(t)_{\mathrm{c}}\right| 0\right\rangle},
$$


где во втором порядке по $\lambda$ имеем

$$
\begin{aligned}
\left\langle 0\left|U(t)_{\mathrm{c}}\right| 0\right\rangle & =(-i \lambda)^{2} \int_{0}^{t} d t_{1} \int_{0}^{t_{1}} d t_{0} \int d p\left|v\left(p_{1}, \ldots, p_{n}\right)\right|^{2} \exp \left(i \sum_{i=1}^{n} \omega\left(p_{i}\right)\left(t_{1}-t_{0}\right)\right)= \\
& =(-i \lambda)^{2} \int_{0}^{t} d t_{0} \int_{0}^{t_{0}} d t_{1} \int d p\left|v\left(p_{1}, \ldots, p_{n}\right)\right|^{2} \exp \left(i \sum_{i=1}^{n} \omega\left(p_{i}\right)\left(t_{1}\right)\right) .
\end{aligned}
$$

Однако согласно методу стационарной фазы

$$
\int d p\left|v\left(p_{1}, \ldots, p_{n}\right)\right|^{2} \exp \left(\sum_{i=1}^{n} \omega\left(p_{i}\right)(t)\right)=O\left(\frac{1}{t^{n d / 2}}\right),
$$

поэтому интеграл

$$
\int_{0}^{+\infty} d t \int d p\left|v\left(p_{1}, \ldots, p_{n}\right)\right|^{2} \exp \left(\sum_{i=1}^{n} \omega\left(p_{i}\right)(t)\right)
$$

при всех $n$ абсолютно сходится и мы можем положить

$$
\left\langle 0\left|U_{\mathrm{c}}(t)\right| 0\right\rangle=A t+B(t),
$$

где

$$
A=(-i \lambda)^{2} \int_{0}^{+\infty} d t \int d p\left|v\left(p_{1}, \ldots, p_{n}\right)\right|^{2} \exp \left(\sum_{i=1}^{n} \omega\left(p_{i}\right)(t)\right)
$$

и

$$
B(t)=\lambda^{2} \int_{0}^{+\infty} d t^{\prime} \min \left(t^{\prime}, t\right) \int d p\left|v\left(p_{1}, \ldots, p_{n}\right)\right|^{2} \exp \left(\sum_{i=1}^{n} \omega\left(p_{i}\right)\left(t^{\prime}\right)\right) .
$$

Теперь покажем, что существует предел $\lim _{t \rightarrow+\infty} B(t)$ Перейдем формально к пределу $t \rightarrow+\infty$ под знаком интеграла в (19) и положим

$$
B:=\lambda^{2} \int_{0}^{+\infty} d t^{\prime} \int d p\left|v\left(p_{1}, \ldots, p_{n}\right)\right|^{2} \exp \left(\sum_{i=1}^{n} \omega\left(p_{i}\right)\left(t^{\prime}\right)\right) .
$$

Чтобы доказать, что этот интеграл сходится в несобственном смысле при $n=$ $1,2, \ldots$, покажем, что

$$
\left.\left|\frac{d^{k}}{d t^{k}} \int d p\right| v\left(p_{1}, \ldots, p_{n}\right)\right|^{2} \exp \left(i \sum_{i=1}^{n} \frac{p_{i}^{2}}{2} t\right) \mid \leqslant \frac{C}{t^{n d / 2+k}}
$$

для некоторой константы $C$. Действительно,

$$
\begin{aligned}
\int d p & \left|v\left(p_{1}, \ldots, p_{n}\right)\right|^{2} \exp \left(\frac{i}{2} \sum_{i=1}^{n} p_{i}^{2} t\right)(x)= \\
= & \int d x F\left(v\left(p_{1}, \ldots, p_{n}\right)\right)^{2}(x) F^{-1}\left(\exp \left(i \sum \frac{p_{i}^{2}}{2} t\right)\right)(x)= \\
= & \frac{1}{(2 \pi)^{n d / 2} t^{n d / 2}} \int F\left(\left|v\left(p_{1}, \ldots, p_{n}\right)\right|^{2}\right)(x) \exp \left(-\frac{i}{2 t} \sum_{i=1}^{n} x_{i}^{2}\right) .
\end{aligned}
$$


Из этого выражения и следует нужная нам оценка. Теперь сходимость интеграла (20) при $\omega \neq 0$ легко показать интегрированием по частям, как это делается при доказательстве критериев Дирихле и Абеля сходимости интегралов.

Рассмотрим разность $B-B(t)$ и покажем, что она стремится к нулю при $t \rightarrow+\infty$. Имеем

$$
B-B(t)=\lambda^{2} t \int_{t}^{+\infty} d t^{\prime} e^{-i \omega n t} \int d p\left|v\left(p_{1}, \ldots, p_{n}\right)\right|^{2} \exp \left(i \sum_{i=1}^{n} \frac{p_{i}^{2}}{2} t^{\prime}\right) .
$$

Снова воспользуемся интегрированием по частям:

$$
\begin{aligned}
B-B(t)=\lambda^{2} t & \frac{e^{-i \omega n t}}{i \omega n} \int d p\left|v\left(p_{1}, \ldots, p_{n}\right)\right|^{2} \exp \left(i \sum_{i=1}^{n} \frac{p_{i}^{2}}{2} t\right)- \\
& -\lambda^{2} t \int_{t}^{+\infty} d t^{\prime} \frac{e^{-i \omega n t^{\prime}}}{i \omega n} \frac{d}{d t} \int d p\left|v\left(p_{1}, \ldots, p_{n}\right)\right|^{2} \exp \left(i \sum_{i=1}^{n} \frac{p_{i}^{2}}{2} t\right) .
\end{aligned}
$$

Пользуясь неравенством (21) для оценки второго интеграла, найдем

$$
|B-B(t)| \leqslant \frac{\text { const }}{t^{n d / 2-1}}
$$

и $B-B(t) \rightarrow 0$, что и утверждалось.

5.2. Пример к теореме 2. Рассмотрим граф Фридрихса $\Phi$, множество вершин которого состоит из трех элементов $\left\{v_{0}, v_{1}, v_{2}\right\}$, множество ребер которого есть $\left\{r_{0}, r_{1}, r_{2}\right\}$, а функции $l^{ \pm}$определены следующими соотношениями: $l^{+}\left(r_{0}\right)=v_{2}$, $l^{-}\left(r_{0}\right)=v_{0}, l^{+}\left(r_{1}\right)=v_{1}, l^{-}\left(r_{1}\right)=v_{0}, l^{+}\left(r_{2}\right)=v_{2}, l^{-}\left(r_{2}\right)=v_{1}$. В обозначениях раздела 3 имеем

$$
J=\int_{1}^{\infty} d \tau_{1} \int_{1}^{\infty} d \tau_{2} \frac{1}{\tau_{1}^{d / 2} \tau_{2}^{d / 2}\left(\tau_{1}+\tau_{2}\right)^{d / 2}}
$$

Воспользуемся неравенством

$$
\left(\tau_{1}+\tau_{2}\right)^{d / 2} \geqslant 2^{d / 2} \tau_{1}^{d / 4} \tau_{2}^{d / 4}
$$

и получим

$$
J \leqslant \operatorname{const} \int_{1}^{\infty} \frac{1}{\tau_{1}^{3 d / 4}} d \tau_{1} \int_{1}^{\infty} \frac{1}{\tau_{1}^{3 d / 4}} d \tau_{2}
$$

Следовательно, интеграл $J$ сходится, если $d>4 / 3$.

С другой стороны, степень расходимости графа $\Phi$ есть $2-3(d / 2)$, а каждой из его поддиаграмм $-1-2(d / 2)$. Таким образом, теорема 2 дает, что интеграл $J$ сходится, если $d>4 / 3$, что совпадает с полученным выше результатом. 
5.3. Выражение коэффициентов $A$ и $B$ через Г-операцию Фридрихса. Пусть

$$
V=\int \prod v\left(p_{1}, \ldots, p_{n} \mid q_{1}, \ldots, q_{m}\right) \prod_{i=0}^{m} a^{+}\left(p_{i}\right) d p_{i} \prod_{j=0}^{m} a\left(q_{j}\right) d q_{j}
$$

- моном Вика. Г-операция Фридрихса определяется следующим образом:

$$
\Gamma(V)=i \int_{-\infty}^{0} d t e^{i t H} V e^{-i t H} .
$$

Легко видеть, что

$$
\Gamma(V)=\int \frac{v\left(p_{1}, \ldots, p_{n} \mid q_{1}, \ldots, q_{m}\right)}{\sum_{i=0}^{n} \omega\left(p_{i}\right)-\sum_{j=0}^{m} \omega\left(q_{j}\right)-i 0} \prod_{i=0}^{n} a^{+}\left(p_{i}\right) d p_{i} \prod_{j=0}^{m} a\left(q_{j}\right) d q_{j} .
$$

Можно показать, что коэффициенты $A$ и $B$ выражаются через Г-операцию Фридрихса следующим образом:

$$
\begin{aligned}
& A=i \sum_{n=2}^{\infty}(-\lambda)^{n}\langle 0|\underbrace{V(\Gamma(V \ldots \Gamma(V) \ldots))}_{n \text { операторов } V}| 0\rangle, \\
& B=-\sum_{n=2}^{\infty}(-\lambda)^{n} \sum_{i=1}^{n}\left\langle 0|\underbrace{\overbrace{\text { о операторов } V}^{V\left(\Gamma \left(V \ldots \Gamma^{2}(V\right.\right.} \ldots \Gamma(V) \ldots)))}_{n \text { операторов } V}| 0\right\rangle .
\end{aligned}
$$

\section{6. ЗАКЛЮЧЕНИЕ}

Мы вывели общую формулу (см. теорему 1), которая описывает поведение на больших временах оператора эволюции. Это формула справедлива для широкого класса гамильтонианов, используемых в квантовой теории поля и статистической механике.

Благодарности. Автор благодарен своему научному руководителю И. В. Воловичу за многочисленные и полезные обсуждения. Работа частично поддержана Российским фондом фундаментальных исследований (грант № 05-01-008884), грантом Президента Российской Федерации (NSh-6705.2006.1) и программой “Современные проблемы теоретической математики” Отделения математических наук РАН.

\section{Список литературы}

[1] Н. Н. Боголюбов, Д. В. Ширков, Введение в теорию квантованных полей, Наука, М., 1976.

[2] Р. Йост, Общая теория квантованных полей, Мир, М., 1967.

[3] М. О. Скалли, М. С. Зубайри, Квантовая оптика, Физматлит, М., 2003.

[4] А. А. Абрикосов, Л. П. Горьков, И. Е. Дзялошинский, Методы квантовой теории поля в статистической физике, Физматгиз, М., 1962.

[5] А. И. Базь, Я. Б. Зельдович, А. М. Переломов, Рассеяние, реакиии и распады в нерелятивистской квантовой механике, Наука, М., 1966. 
[6] Л. Б. Окунь, Физика элементарных частии, Наука, М., 1988.

[7] L. Accardy, Yu. G. Lu, I. V. Volovich, Quantum Theory and Its Stochastic Limit, Springer, Berlin, 2002.

[8] A. N. Pechen, I. V. Volovich, Infin. Dimens. Anal. Quantum Probab. Relat. Top., 5:4 (2002), 441-464; math-ph/0202046.

[9] I. Ya. Aref'efa, I. V. Volovich, Infin. Dimens. Anal. Quantum Probab. Relat. Top., 3:4 (2000), 453-482; Quantum decoherence and higher order corrections to the large time exponential behaviour, quant-ph/9906022.

[10] К. Хепп, Теория перенормировок, Наука, М., 1974.

[11] О.И. Завьялов, Перенормированные диаграммы Фейнмана, Наука, М., 1979; В. А. Смирнов, Перенормировка и асимптотические разложения фейнмановских амплитуд, Изд-во МГУ, М., 1990; А. N. Kuznetsov, F. V. Tkachov, V. V. Vlasov, Techniques of Distributions in Perturbative Quantum Field Theory, (I) Euclidean asymptotic operation for products of singular functions, hep-th/9612037.

[12] П. П. Кулиш, Л. Д. Фаддеев, ТМФ, 4 (1970), 153-170.

[13] Л. Д. Фаддеев, ДАН СССР, 152 (1963), 573-576.

[14] I. Ya. Aref'eva, TMФ, 14 (1973), 3-17.

[15] J. R. Dorfman, E. G. D. Cohen, J. Math. Phys., 8:2 (1967), 282-297; J. R. Dorfman, Physica A, 106 (1981), 77-101.

[16] H. Saller, The Hilbert spaces for stable and unstable particles, hep-th/0501074.

[17] Yong Zhou, A new wave-function renormalization prescription for unstable particle, hep-ph/0510244.

[18] L. Maiani, M. Testa, Ann. Phys., 263 (1998), 353-367; hep-th/9709110.

[19] P. Caban, J. Rembieliński, K. A. Smoliński, Z. Walczak, Phys. Rev. A, 72 (2005), 032106; quant-ph/0506183.

[20] К. Фридрихс, Возмущение спектра операторов в гилъбертовом пространстве, Мир, М., 1969.

[21] Ф.П. Васильев, А. Ю. Иваницкий, Линейное программирование, Факториал, М., 1998.

[22] В. С. Владимиров, Уравнения математической физики, Наука, М., 1981. 